\title{
Monitoramento da cobertura vegetal por sensoriamento remoto no semiárido brasileiro através de índices de vegetação
}

\section{Caio Victor Santos SILVA ${ }^{1}$, Jhon Lennon Bezerra da SILVA ${ }^{1 *}$, Geber Barbosa de Albuquerque MOURA ${ }^{1}$, Pabrício Marcos Oliveira LOPES ${ }^{1}$, Cristina Rodrigues NASCIMENTO ${ }^{1}$, Landson Carlos da SILVA ${ }^{1}$}

${ }^{1}$ Universidade Federal Rural de Pernambuco, Recife, PE, Brasil.

*E-mail: jhonlennonsilva@yahoo.com.br

\begin{abstract}
Recebido em dezembro/2018; Aceito em março/2019.
RESUMO: São necessárias medidas que visem à proteção e conservação dos recursos hídricos e naturais de forma rápida e eficiente. As técnicas de sensoriamento remoto são essenciais para o monitoramento ambiental dos recursos no semiárido no espaço e no tempo. Objetivou-se monitorar e analisar à dinâmica da cobertura vegetal através da variabilidade espaço-temporal do albedo da superfície e índices de vegetação em região de Caatinga do semiárido brasileiro por sensoriamento remoto. A área de estudo é o município de Arcoverde, localizado no semiárido de Pernambuco. O estudo foi desenvolvido através de seis imagens orbitais do Landsat5 do sensor TM. O processamento digital dos parâmetros biofísicos foi realizado pelo algoritmo SEBAL. Os resultados foram analisados através da estatística descritiva e quanto a sua variabilidade. Áreas possivelmente degradadas foram identificadas pelos altos valores de albedo e índices de vegetação significativamente menores, localizadas à sudoeste e noroeste da região. Os índices apresentaram comportamento similares, principalmente no período seco, com baixos valores sendo próximos de zero, áreas afetadas pelo período de seca no semiárido. O SAVI apresentou maior precisão, destacando melhor resposta espectral da vegetação. O sensoriamento remoto promoveu monitoramento espaço-temporal adequado, destacando principalmente o período classificado como climaticamente seco através do albedo e índices de vegetação.
\end{abstract}

Palavras-chave: Caatinga; NDVI; SAVI; mudanças ambientais; SEBAL.

\section{Monitoring of vegetation cover by remote sensing in Brazilian semiarid through vegetation indices}

\begin{abstract}
Measures are needed aimed at the protection and conservation of water and natural resources quickly and efficiently. Remote sensing techniques are essential for the environmental monitoring of resources in the semiarid region in space and time. Aimed to monitor and analyze the dynamics of vegetation cover through the spatial-temporal variability of the surface albedo and indices of vegetation in the Caatinga region of the Brazilian semiarid by remote sensing. The study area is the municipality of Arcoverde, located in the semiarid of Pernambuco. The study was developed through six orbital images of Landsat-5 of the TM sensor. The digital processing of the biophysical parameters was performed by the SEBAL algorithm. The results were analyzed through descriptive statistics and their variability. Possibly degraded areas were identified by high albedo values and significantly lower vegetation indices, located in the southwest and northwest of the region. The indexes showed similar behavior, mainly in the dry period, with low values being close to zero, areas affected by the dry period in the semiarid. The SAVI presented higher accuracy, highlighting better spectral response of the vegetation. Remote sensing promoted adequate space-time monitoring, highlighting mainly the period classified as climatically dry through the albedo and vegetation indexes.
\end{abstract}

Keywords: Caatinga; NDVI; SAVI; environmental changes; SEBAL.

\section{INTRODUÇÃO}

O semiárido brasileiro encontra-se sobre um cenário de mudanças na sua cobertura vegetal de Caatinga e, principalmente com a problemática da escassez de água, vivenciada fortemente nas últimas décadas. Os longos períodos de seca influenciaram severamente as principais deficiências nessas regiões, favorecendo mais comumente o aparecimento de áreas em processo de degradação e/ou degradadas, o que futuramente por consequência podem tornar-se áreas desertificadas. As atividades antrópicas também são responsáveis e aceleram esses processos, sendo as que mais causam o aquecimento global e, consequentemente as mudanças climáticas e a queima de combustíveis fósseis, atividades industriais e transporte, conversão do uso do solo, agropecuária, descarte de resíduos sólidos e o desmatamento (RIBEIRO et al., 2016; OLIVEIRA et al., 2017; MARIANO et al., 2018; WWF, 2018).

A Caatinga, bioma que ocupa cerca de $11 \%$ do território nacional brasileiro $\left(844.453 \mathrm{~km}^{2}\right)$ é o principal ecossistema da Região Nordeste do Brasil e Norte de Minas Gerais, trata-se do bioma semiárido mais biodiverso do mundo, sempre foi decisiva para a economia da região, fornece $30 \%$ de sua matriz energética oriunda da vegetação, além de produtos florestais não madeireiros fundamentais para geração de emprego e renda da população, que chega a 27 milhões de habitantes, entretanto estas atividades são realizadas de modo predatório 
contribuindo para o desmatamento que chega a 46\% da área do bioma (MMA, 2018).

A partir deste cenário, alerta-se para a necessidade de medidas que visem à proteção e conservação dos remanescentes de vegetação natural de Caatinga, sendo essencial a observação dos padrões da cobertura vegetal nas regiões semiáridas, que possibilitem o monitoramento de alterações sobre os usos do solo. No Brasil o Projeto de Conservação e Utilização Sustentável da Diversidade Biológica Brasileira (PROBIO) utilizou técnicas de sensoriamento remoto para o mapeamento da cobertura vegetal dos biomas brasileiros, o que permitiu ao Instituto Brasileiro do Meio Ambiente e dos Recursos Naturais (IBAMA) realizar o monitoramento da Caatinga identificando áreas desmatadas entre o período de 2002 a 2009 (MMA, 2011). Constantemente estudos neste sentido estão sendo desenvolvidos para análise da vegetação no semiárido brasileiro, no intuito de monitorar e avaliar o grau de degradação ambiental e desertificação do solo (RODRIGUES et al., 2009; ARRAES et al., 2012; BEZERRA et al., 2014; SILVA et al., 2016; SILVA et al., 2017).

Nas últimas décadas, o geoprocessamento tem-se destacado fortemente no desenvolvimento de geotecnologias e aberto um novo campo de atuação do monitoramento ambiental. Em especial, as tecnologias envolvidas na detecção de respostas espectrais em sensores aerotransportados, como o sensoriamento remoto, ferramenta de alta capacidade e responsável pelo monitoramento ambiental em larga escala dos diferentes usos do solo no espaço e no tempo com eficácia, permitindo medidas de reversão de forma rápida e à baixo custo, por meio do planejamento e tomada de decisões para o uso sustentável dos recursos hídricos e naturais (BEZERRA et al., 2014; GIONGO; VETTORAZZI, 2014; SILVA et al., 2017).

Dentre as aplicações mais utilizadas no sensoriamento remoto, destacam-se os estudos sobre a detecção de mudanças e monitoramento da cobertura vegetal (RÊGO et al., 2012; SILVA; GALVÍNCIO, 2012; OLIVEIRA et al., 2017), e entre as principais análises atribuídas a este segmento, as aplicações de índices de vegetação são importantes para distinguir as informações espectrais da vegetação dos outros elementos da superfície terrestre, além de indicar a quantidade e qualidade da vegetação observada em determinadas épocas do ano (RIBEIRO et al., 2016; LINS et al., 2017).

Diante deste contexto, objetivou-se monitorar e analisar a dinâmica da cobertura vegetal através da variabilidade espaçotemporal do albedo da superfície e índices de vegetação em região de Caatinga do semiárido brasileiro, utilizando técnicas de sensoriamento remoto com uso de imagens orbitais de satélite em períodos seco, normal e chuvoso.

\section{MATERIAL E MÉTODOS}

\section{1. Área de estudo}

Selecionou-se para a presente pesquisa, o município de Arcoverde, localizado entre os paralelos de $8^{\circ} 16^{\prime} 35,97^{\prime}$ ' S (UTM: 9084500 m) e 8० 31' 19,18', S (UTM: 9057500 m), e entre os meridianos de $36^{\circ} 53^{\prime} 21,11^{\prime}$ 'W (UTM: $732500 \mathrm{~m}$ ) e $37^{\circ} 08^{\prime} 15,36$ ' W (UTM: $705000 \mathrm{~m}$ ), altitude de $663 \mathrm{~m}$, região semiárida de Pernambuco, mais especificamente, está inserido na Mesorregião do Sertão Pernambucano e na Microrregião do Sertão do Moxotó, Região Nordeste do Brasil. O município abrange uma área de $323,369 \mathrm{~km}^{2}$ com altitudes variando entre 650 e 1.000 m (IBGE, 2018).
A região possui zona climática do tipo BSh (Clima semiárido quente) conforme classificação de Köppen, com verão seco e período chuvoso compreendido entre os meses de janeiro a setembro, podendo estender-se até outubro. A precipitação pluviométrica média anual é de $653 \mathrm{~mm}$ e as temperaturas médias variam entre 12 e $25^{\circ} \mathrm{C}$ de acordo com os registros pluviométricos do Instituto Nacional de Meteorologia (INMET) dos últimos 30 anos (1987-2016) disponibilizados pela Agencia Pernambucana de Águas e Clima (APAC) (ALVARES et al., 2013; LINS et al., 2017). Apresenta ainda, vegetação característica de áreas de agreste e sertão, formada por florestas subcaducifólias e caducifólias (BELTRÃO et al., 2005).

A Figura 1 apresenta a área de estudo em composição colorida, através de uma imagem orbital do satélite Landsat. Destacando-se os pontos de localização da cidade e estação meteorológica responsável pelos registros de precipitação pluviométrica.
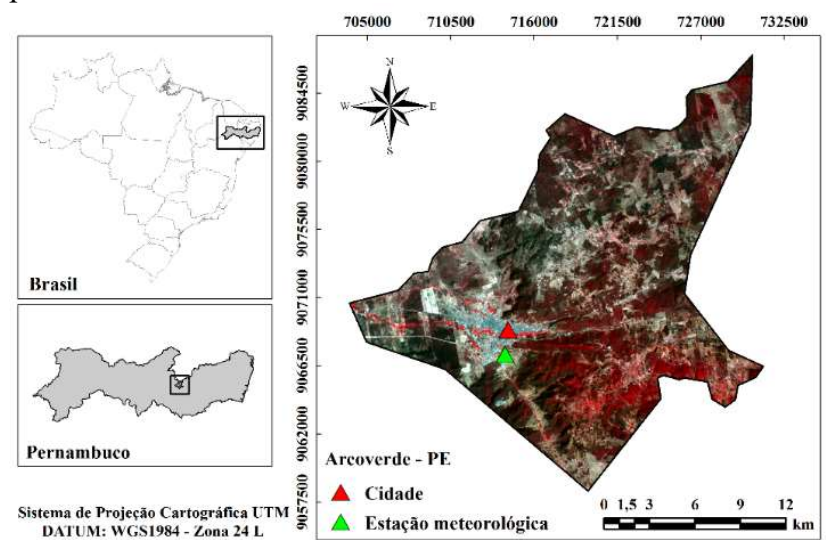

DATUM: WGS1984 - Zona 24 L
DAT Projecã Cartográica UT

Figura 1. Mapa espacial de localização da área de estudo: município de Arcoverde, Pernambuco, Brasil.

Figure 1. Spatial map of location of study area: Arcoverde municipality, Pernambuco, Brazil.

\subsection{Regimes de precipitação pluviométrica}

Através da série histórica de 1984 a 2014, um período de 31 anos consecutivos de acúmulo anual da precipitação pluviométrica $(\mathrm{P}, \mathrm{mm})$ registrados mensalmente pela estação automática do INMET foi realizado a técnica estatística dos Quantis, para classificação dos anos secos, normais e chuvosos da região conforme a metodologia de Xavier; Xavier (1999). Configurou-se climaticamente como sendo anos secos, os de 2003 e 2006 (Figura 2), anos normais os de 2007 e 2008 (Figura 3) e chuvosos foram os anos 2004 e 2010 (Figura 4).

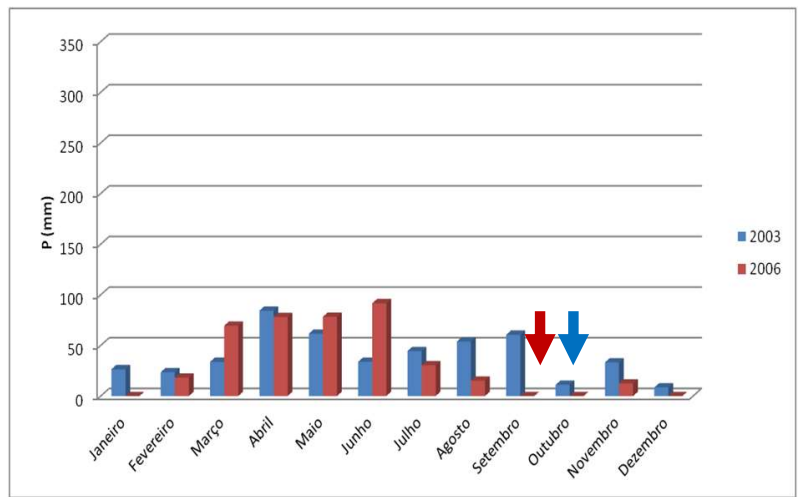

Figura 2. Regimes pluviométricos dos anos secos de 2003 e 2006 para o município de Arcoverde-PE.

Figure 2. Pluviometric regimes of the dry years of 2003 and 2006 for the municipality of Arcoverde-PE. 


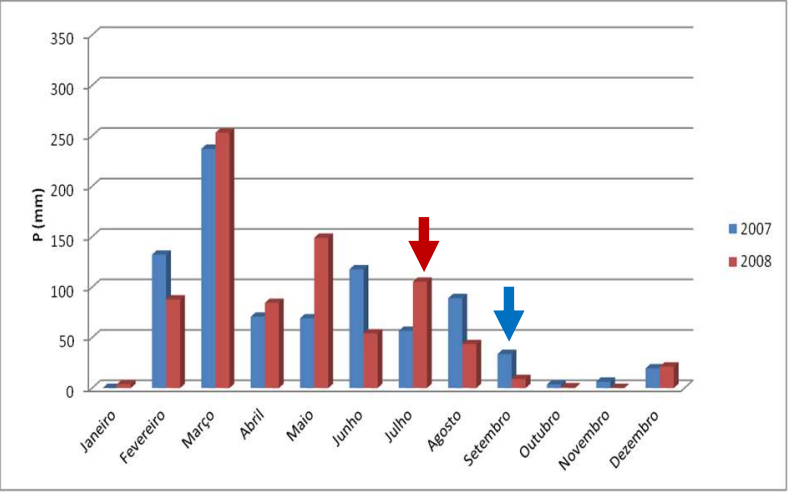

Figura 3. Regimes pluviométricos dos anos normais de 2007 e 2008 para o município de Arcoverde-PE.

Figure 3. Pluviometric regimes of the normal years of 2003 and 2006 for the municipality of Arcoverde-PE.

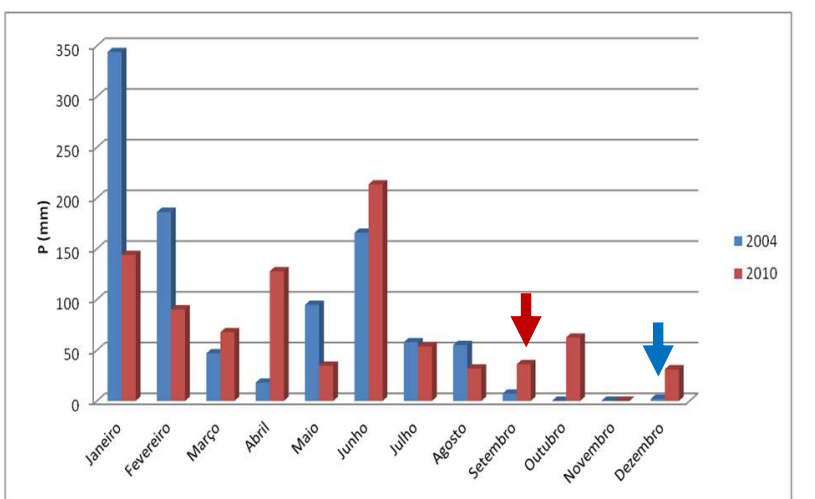

Figura 4. Regimes pluviométricos dos anos chuvosos de 2004 e 2010 para o município de Arcoverde-PE.

Figure 4. Pluviometric regimes of the rainy years of 2003 and 2006 for the municipality of Arcoverde-PE.

A Tabela 1 destaca os registros de precipitação do trimestre anterior, bem como, no mesmo dia do imageamento pelo satélite e, também, apresenta o total médio anual de cada ano classificado como seco, normal e chuvoso.

\subsection{Dados orbitais de satélite}

O estudo foi desenvolvido através do uso de seis imagens orbitais do satélite Landsat-5 do sensor TM, nos dias: $12 / 10 / 2003$ e 17/12/2004, 18/09/2006, 19/07/2007,
23/09/2008 e 29/09/2010, adquiridas através do Serviço Geológico dos Estados Unidos - USGS (United States Geological Survey) pertencente a base de dados espaciais americana da NASA (National Aeronautics and Space Administration). As imagens foram escolhidas em conformidade com anos classificados como climaticamente secos (nos dias 12/10/2003 e 18/09/2006), normais (nos dias $19 / 07 / 2007$ e $23 / 09 / 2008$ ) e chuvosos (nos dias 17/12/2004 e 29/09/2010).

Tabela 1. Registros da precipitação pluviométrica dos últimos três meses anterior ao imageamento pelo satélite na região semiárida. Table 1. Rainfall records of the last three months prior to satellite imagery in the semiarid region.

\begin{tabular}{ccccc}
\hline \multirow{2}{*}{$\begin{array}{c}\text { Classificação } \\
\text { dos anos }\end{array}$} & Data & $\begin{array}{c}\text { Trimestre } \\
\text { anterior }\end{array}$ & $\begin{array}{c}\text { Mesmo } \\
\text { dia }\end{array}$ & $\begin{array}{c}\text { Total } \\
\text { médio } \\
\text { anual }\end{array}$ \\
\hline \multirow{2}{*}{ Seco } & $12 / 10 / 2003$ & 158,7 & 11 & 474,7 \\
& $18 / 09 / 2006$ & 137,1 & 0 & 393,2 \\
\hline \multirow{2}{*}{ Normal } & $19 / 07 / 2007$ & 275,5 & 56,7 & 835,7 \\
& $23 / 09 / 2008$ & 203 & 9 & 811,8 \\
\hline \multirow{2}{*}{ Chuvoso } & $17 / 12 / 2004$ & 7,4 & 2 & 977,2 \\
& $29 / 09 / 2010$ & 298,9 & 36,1 & 892,7 \\
\hline
\end{tabular}

O processamento digital e semi-automatizado das imagens foi realizado através do Software ERDAS IMAGINE® 9.1, com a implementação do algoritmo SEBAL (Surface Energy Balance Algorithm for Land). A metodologia do SEBAL foi conforme Bastiaanssen (2000) e Allen et al. (2002). O algoritmo foi responsável pelas operações matemáticas de processamento digital, onde converteu-se os níveis de cinza das imagens em radiância espectral e refletância monocromática para estimativas de parâmetros biofísicos como o albedo da superfície e índices de vegetação.

A Tabela 2 apresenta os coeficientes de calibração (radiância mínima $-\mathrm{L}_{\text {mín }}$; radiância máxima $-\mathrm{L}_{\text {máx } 1} \mathrm{e} \mathrm{L}_{\text {máx }}$, onde para o período de maio/2003 a abril/2007 utilizou-se os

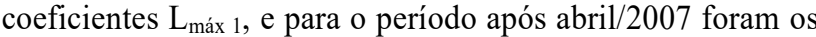
coeficientes $\mathrm{L}_{\text {máx }}$ 2), também se destaca as irradiâncias espectrais de cada banda $\left(\mathrm{k}_{\mathrm{b}}\right)$ no topo da Atmosfera (TOA) para cada banda do satélite Landsat-5 TM, propostos por Chander et al. (2009).

Tabela 2. Valores dos coeficientes de calibração radiométrica do Landsat-5 TM.

Table 2. Values of radiometric calibration coefficients of the Landsat-5 TM.

\begin{tabular}{|c|c|c|c|c|c|}
\hline \multirow[t]{2}{*}{ Banda } & \multirow{2}{*}{$\begin{array}{l}\text { Comprimento de ondas } \\
\qquad(\mu \mathrm{m})\end{array}$} & \multicolumn{3}{|c|}{$\begin{array}{l}\text { Coeficientes de calibração do } \\
\text { Landsat-5 TM }\left(\mathrm{Wm}^{-2} \mathrm{sr}^{-1} \mu \mathrm{m}^{-1}\right)\end{array}$} & \multirow{2}{*}{$\begin{array}{l}\text { Irradiância espectral }\left(\mathrm{k}_{\mathrm{b}}\right) \text { no topo da } \\
\text { Atmosfera (TOA, } \mathrm{W} \mathrm{m}^{-2} \mu^{-1} \text { ) }\end{array}$} \\
\hline & & $\mathrm{L}_{\min }$ & $\mathrm{L}_{\max 1}$ & $\mathrm{~L}_{\max 2}$ & \\
\hline 1 (azul) & $0,45-0,52$ & $-1,52$ & 193 & 169 & 1957 \\
\hline 2 (verde) & $0,52-0,60$ & $-2,84$ & 365 & 333 & 1796 \\
\hline 3 (vermelho) & $0,63-0,69$ & $-1,17$ & 264 & 264 & 1536 \\
\hline 4 (IV-próximo) & $0,76-0,90$ & $-1,51$ & 221 & 221 & 1031 \\
\hline 5 (IV-médio) & $1,55-1,75$ & $-0,37$ & 30,2 & 30,2 & 220 \\
\hline 6 (IV-termal) & $10,40-12,50$ & 1,2378 & 15,303 & 15,303 & - \\
\hline 7 (IV-médio) & $2,08-2,35$ & $-0,15$ & 16,5 & 16,0 & 83,44 \\
\hline
\end{tabular}

*IV - Infravermelho.

Para calibração de cada banda utilizou-se seus respectivos coeficientes na conversão dos números digitais (ND) em radiância espectral, pois os satélites medem tais radiâncias emitidas e refletidas pelos alvos e armazenam os dados em formato digital conforme a Equação 1 (ALLEN et al., 2002; CHANDER et al., 2009).

$$
\mathrm{L}_{\mathrm{b}}=\mathrm{L}_{\text {mín b }}+\left(\frac{\mathrm{L}_{\text {máx b }}-\mathrm{L}_{\text {mín b }}}{255}\right) \cdot\left(\mathrm{ND}_{\mathrm{b}}-1\right)
$$

em que: $\mathrm{L}_{b}\left(\mathrm{~W} \mathrm{~m}^{-2} \mathrm{sr}^{-1} \mu \mathrm{m}^{-1}\right)$ é a radiância espectral em cada banda;

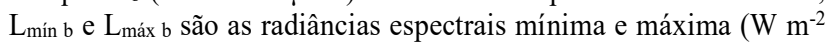
$\left.\mathrm{sr}^{-1} \mu \mathrm{m}^{-1}\right)$ e $\mathrm{ND}_{\mathrm{b}}$ a intensidade do pixel (número digital de 0 a 256 ). 
A refletância monocromática foi determinada em função do ângulo zenital solar $(\theta)$ e o inverso do quadrado da distância relativa Terra-Sol $\left(\mathrm{d}_{\mathrm{r}}\right)$ conforme a Equação 2 (ALLEN et al., 2002; CHANDER et al., 2009).

$$
\mathrm{r}_{\mathrm{b}}=\frac{\pi \cdot \mathrm{L}_{\mathrm{b}}}{\mathrm{k}_{\mathrm{b}} \cdot \cos \theta \cdot \mathrm{d}_{\mathrm{r}}}
$$

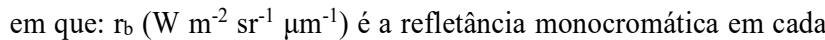
banda; $\mathrm{kb}_{\mathrm{b}}\left(\mathrm{W} \mathrm{m}^{-2} \mu \mathrm{m}^{-1}\right)$ é a irradiância solar espectral de cada uma das bandas refletivas do Landsat-5 TM no topo da Atmosfera e $\theta$ é o ângulo zenital solar. 1983)

$O \mathrm{~d}_{\mathrm{r}}$ foi determinado conforme a Equação 3 (IQBAL,

$\mathrm{d}_{\mathrm{r}}=1+0,033 \cdot \cos \left(\frac{\mathrm{DSA} \cdot 2 \cdot \pi}{365}\right)$

em que: DSA é o dia sequencial do ano e o argumento da função cos está em radianos.

O ângulo zenital solar $(\theta)$ foi obtido através do ângulo de elevação do Sol (E, graus) conforme Equação 4.

$$
\theta=\cos \left(\frac{\pi}{2}-E\right)=\operatorname{sen}(E)
$$

Através das refletâncias de cada banda $\left(\mathrm{r}_{1}, \mathrm{r}_{2}, \mathrm{r}_{3}, \mathrm{r}_{4}, \mathrm{r}_{5}\right.$ e $\left.\mathrm{r}_{7}\right)$ do Landsat-5 TM determinou-se o albedo planetário conforme a Equação 5 (ALLEN et al., 2002; SILVA et al., 2005b).

$\alpha_{\text {toa }}=0,293 \cdot r_{1}+0,274 . r_{2}+0,233 . r_{3}+0,157 . r_{4}+0,033$

$\mathrm{r}_{5}+0,011 \cdot \mathrm{r}_{7}$

em que: 0,$293 ; 0,274 ; 0,233 ; 0,157 ; 0,033$ e 0,011 são coeficientes de pesos propostos pela NASA para o Landsat-5 TM, utilizados com frequência em estudos no semiárido brasileiro (SILVA et al., 2005b; BEZERRA et al., 2014). São encontrados através dos valores das irradiâncias espectrais $(\mathrm{kb})$ de cada banda (Tabela 2) pela somatória das irradiâncias de todas as bandas $\left(\Sigma \mathrm{k}_{\mathrm{b}}\right)$.

Portanto, o albedo corrigido da superfície foi computado de acordo com vários estudos no mundo e, principalmente no semiárido brasileiro conforme a Equação 6 (BASTIAANSSEN, 2000; ALLEN et al., 2002; SILVA et al., 2005a; SILVA et al., 2016; LINS et al., 2017).

$$
\alpha_{\text {sup }}=\frac{\alpha_{\text {toa }-} \alpha_{\text {atm }}}{\tau_{\text {sw }}^{2}}
$$

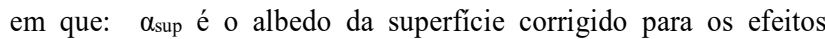

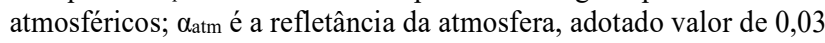
conforme as pesquisas de Bastiaanssen (2000) e Silva et al. (2005a) e $\tau_{\text {sw }}$ é a transmissividade atmosférica no domínio da radiação solar para dias de céu claro conforme a Equação 7 (ALLEN et al., 2002).

$$
\tau_{\mathrm{sw}}=0,35+0,627 . \exp \left[\frac{-0,00146 \cdot \mathrm{P}_{\mathrm{o}}}{\mathrm{K}_{\mathrm{t}} \cdot \cos \theta}-0,075\left(\frac{\mathrm{W}}{\cos \theta}\right)^{0,4}\right]
$$

em que: $P_{o}(\mathrm{kPa})$ é a pressão atmosférica instantânea; $K_{t}$ é o coeficiente de turbidez da atmosfera no valor de 1,0 (céu claro e limpo) conforme Allen et al. (2007); W (mm) é a água precipitável obtida em função da umidade relativa do ar instantânea obtida conforme a Equação 8 (GARRISON; ADLER 1990).

$$
\mathrm{W}=0,14 \cdot \mathrm{e}_{\mathrm{a}} \cdot \mathrm{P}_{\mathrm{o}}+2,1
$$

em que: $e_{a}(\mathrm{kPa})$ é a pressão real de vapor obtida conforme a Eq. 9 .

$$
\mathrm{e}_{\mathrm{a}}=\frac{\mathrm{UR} \cdot \mathrm{e}_{\mathrm{s}}}{100}
$$

em que: $\mathrm{e}_{\mathrm{s}}(\mathrm{kPa})$ é a pressão de saturação obtida conforme a Equação 10.

$$
\mathrm{e}_{\mathrm{s}}=0,6108 \cdot \exp \left(\frac{17,27 \cdot \mathrm{T}_{\mathrm{ar}}}{237,3+\mathrm{T}_{\mathrm{ar}}}\right)
$$

em que: $\mathrm{T}_{\text {ar }}\left({ }^{\circ} \mathrm{C}\right)$ é a temperatura do ar instantânea.

Também através das bandas refletivas, determinou-se os índices de vegetação NDVI, SAVI e IAF. O índice de vegetação da diferença normalizada (NDVI) é um indicador sensível da condição da vegetação verde/natural ou agrícola. É um índice bastante utilizado nas aplicações com lavouras e estimativas de produtividade, detecção dos efeitos de secas, mapeamento de áreas agrícolas, etc. Possui variação entre -1 e 1 . Onde quanto mais próximo de 1 , maior é a indicação da atividade vegetativa, ou seja, onde há forte atividade clorofiliana. Valores negativos indicam áreas de corpos hídricos. Valores próximos e na faixa de zero indicam áreas com pouca ou nenhuma vegetação, ou seja, onde há pouca ou nenhuma atividade clorofiliana (BEZERRA et al., 2014), determinado conforme a Equação 11 (ALLEN et al., 2002).

$$
\mathrm{NDVI}=\frac{\mathrm{r}_{\mathrm{b} \text { IV }}-\mathrm{r}_{\mathrm{bV}}}{\mathrm{r}_{\mathrm{b} \text { IV }}+\mathrm{r}_{\mathrm{bV}}}
$$

em que: $r_{b}$ IV e $r_{b} v$, correspondem às respectivas bandas refletivas $4 \mathrm{e}$ 3 do Landsat-5 de sensor TM. São as bandas do infravermelho próximo e vermelho (Tabela 2 ).

O Índice de vegetação ajustado ao solo (SAVI) é um parâmetro que busca amenizar os efeitos antecedentes do solo através de um fator de ajuste dependente do tipo de solo da área de estudo (RIBEIRO et al., 2016). A aplicação deste índice ameniza limitações impostas pelo NDVI. O SAVI foi determinado de acordo com a Equação 12 (ALLEN et al., 2002).

$$
\mathrm{SAVI}=\frac{(1+\mathrm{L}) \cdot\left(\mathrm{r}_{\mathrm{b} \text { IV }}-\mathrm{r}_{\mathrm{bV}}\right)}{\left(\mathrm{L}+\mathrm{r}_{\mathrm{bIV}}+\mathrm{r}_{\mathrm{bV}}\right)}
$$

em que: L é a constante de ajuste ao solo, que varia entre 0 e 1 conforme a densidade da cobertura vegetal local. O valor 1 é utilizado para áreas com pouca vegetação. Valor de 0,5 são para áreas com cobertura vegetal intermediária. Valor de 0,25 é para áreas de coberturas muito densas e zero torna o índice SAVI semelhante ao NDVI (HUETE, 1988; ALLEN et al., 2007). Para o presente estudo utilizou-se o fator de ajuste 0,5 , visando minimizar erros devido a região se encontrar no semiárido e possuir vegetação de Caatinga (SILVA; GALVÍNCIO, 2012).

O índice SAVI foi utilizado para determinação do índice de área foliar (IAF, $\mathrm{m}^{2} \mathrm{~m}^{-2}$ ). O IAF é um indicador da biomassa vegetal, representado pela razão entre a área foliar do dossel e a unidade de superfície projetada no solo, conforme a Equação 13 (ALLEN et al., 2007).

$$
\mathrm{IAF}=-\frac{\ln \left(\frac{0,69-\mathrm{SAVI}}{0,59}\right)}{0,91}
$$

O índice IAF varia entre 0 e $10 \mathrm{~m}^{2} \mathrm{~m}^{-2}$, desde áreas com pouca ou nenhuma vegetação a áreas densas. Quanto menor for a atividade fotossintética, menor será o valor do IAF. 


\subsection{Análise estatística}

Os resultados foram submetidos a medidas de tendência central (mínimo - Min, máximo - Max, médio, mediano, moda) e dispersão (desvio padrão - DP, variância e coeficiente de variação - CV). Também foi avaliado o CV (\%) quanto a sua variabilidade conforme os critérios de classificação de Warrick; Nielsen (1980), sendo CV $<12 \%$ (baixa variabilidade), $\mathrm{CV}$ entre 12 e $60 \%$ (média variabilidade) e $\mathrm{CV}$ $>60 \%$ (alta variabilidade).

\section{RESULTADOS}

A Figura 5 destaca a variabilidade espaço-temporal do albedo da superfície, distinguindo tonalidades conforme as especificidades de cada pixel sobre os diferentes usos e ocupações do solo na região semiárida de Arcoverde-PE. A análise do albedo variou entre 0 e 0,70 e foi incorporada no intuito de verificar com mais acurácia e, também, para confirmar as principais alterações ambientais da região semiárida a serem destacadas nos índices de vegetação.

A Figura 6 apresenta a variabilidade espaço-temporal do índice de vegetação NDVI, responsável por destacar tanto a quantidade como a condição verde da cobertura vegetal (BEZERRA et al., 2014). O NDVI variou entre -1 e 1, sendo importante frisar que os valores negativos foram diante da presença de corpos hídricos (pixel azul), já para os valores próximos de zero e/ou zero indicaram áreas com pouca vegetação e/ou solo exposto (pixel amarelo) e os altos valores foram sobre áreas bem vegetadas e/ou áreas irrigadas (pixel verde).

A Figura 7 apresenta a variabilidade espaço-temporal do índice de vegetação SAVI, é um bom indicador da biomassa de cada pixel nos mapas temáticos (ALLEN et al., 2002). O SAVI também variou entre -1 e 1 e apresentou comportamento similar ao NDVI. Os valores negativos foram observados sobre a superfície de água (pixel azul). Valores do SAVI em zero e/ou próximos de zero foram sobre áreas com solo exposto e/ou pouca vegetação (pixel amarelo), já os maiores valores foram sobre áreas irrigadas e vegetação de Caatinga densa (pixel verde).

A Figura 8 apresenta a variabilidade espaço-temporal do índice de vegetação IAF, que variou de $0 \mathrm{a}>1 \mathrm{~m}^{2} \mathrm{~m}^{-2}$. O IAF, assim como foi nos outros índices, destacou a dinâmica espaço-temporal da região semiárida observando valores menores sobre áreas com pouca ou nenhuma vegetação (pixel vermelho), ou seja, valores próximos de zero, confirmando junto aos índices NDVI e SAVI o significativo aumento de áreas de solo exposto na região, sendo possivelmente áreas degradadas. Os maiores valores do IAF foram representados principalmente sobre as áreas com vegetação de Caatinga densa (pixel verde).

Em destaque na Tabela 3, pode-se observar as análises estatísticas dos principais resultados dos índices de vegetação NDVI, SAVI e IAF. Apresentam-se valores em termos mínimo, máximo, média, mediana, moda, desvio padrão e coeficiente de variação.

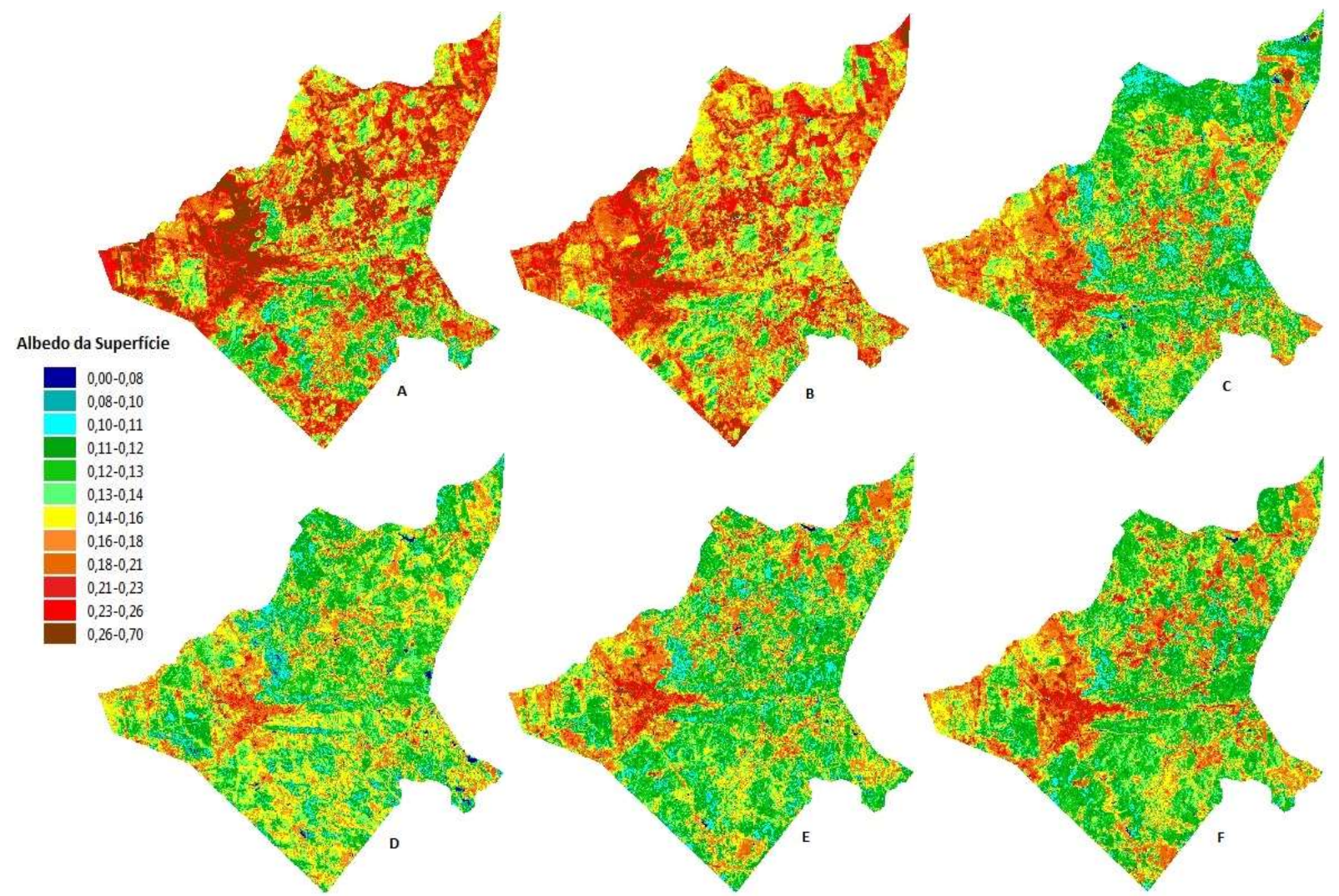

Figura 5. Variabilidade espaço-temporal do albedo da superfície nos dias 12/10/2003 (A), 18/09/2006 (B) (período seco); 19/07/2007 (C), 23/09/2008 (D) (período normal); 17/12/2004 (E) e 29/09/2010 (F) (período chuvoso) para o município de Arcoverde - PE.

Figure 5. Spatial-temporal variability of the surface albedo in the days 10/12/2003 (A), 09/18/2006 (B) (dry period); 07/19/2007 (C), 09/23/2008 (D) (normal period); 12/17/2004 (E) and 09/29/2010 (F) (rainy season) for the municipality of Arcoverde - PE. 
Silva et al.

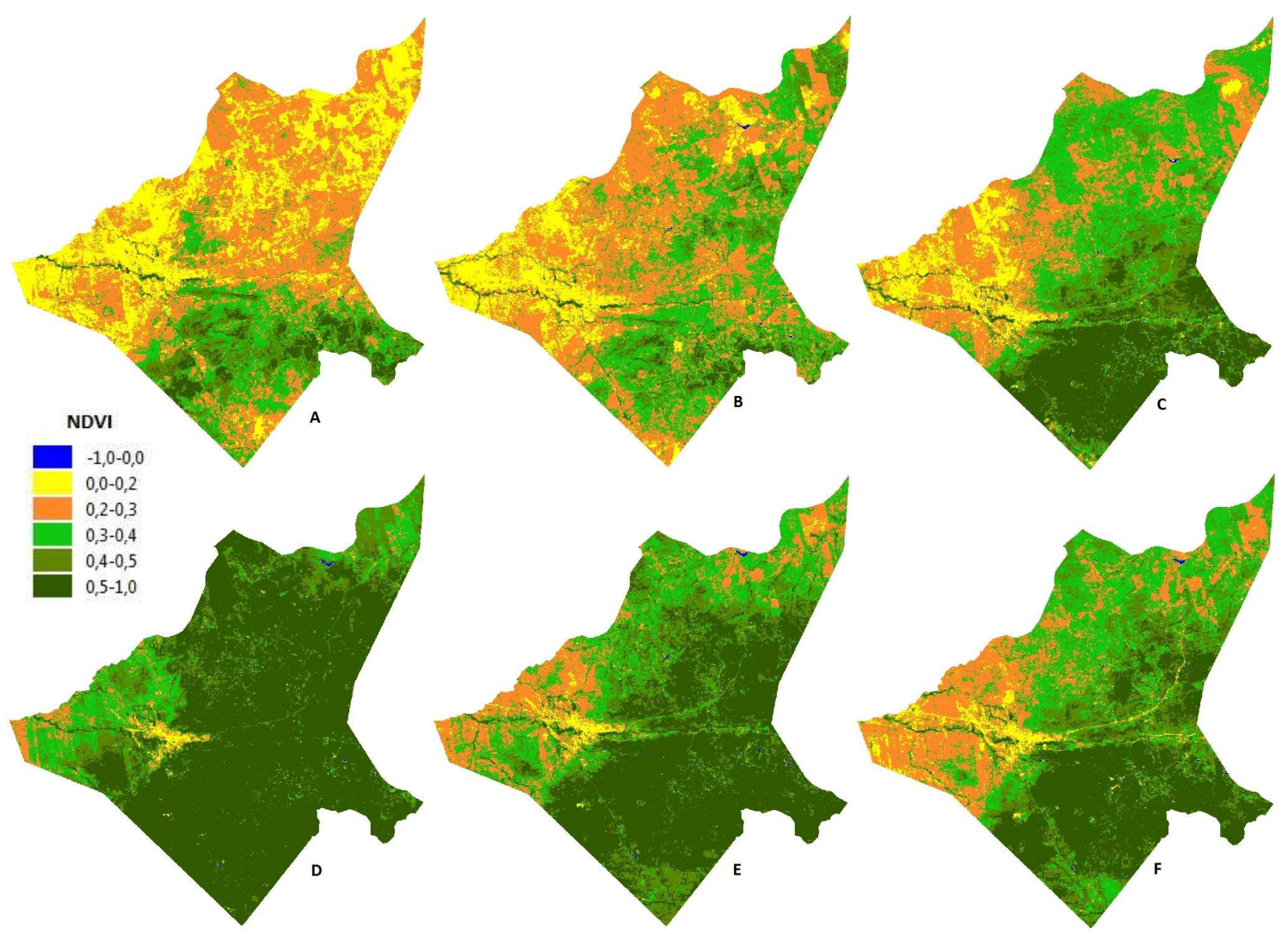

Figura 6. Variabilidade espaço-temporal do índice de vegetação NDVI nos dias 12/10/2003 (A), 18/09/2006 (B) (período seco); 19/07/2007 (C), 23/09/2008 (D) (período normal); 17/12/2004 (E) e 29/09/2010 (F) (período chuvoso) para o município de Arcoverde - PE.

Figure 6. Spatial-temporal variability of NDVI vegetation index in the days 10/12/2003 (A), 09/18/2006 (B) (dry period); 07/19/2007 (C), 09/23/2008 (D) (normal period); 12/17/2004 (E) and 09/29/2010 (F) (rainy season) for the municipality of Arcoverde - PE.

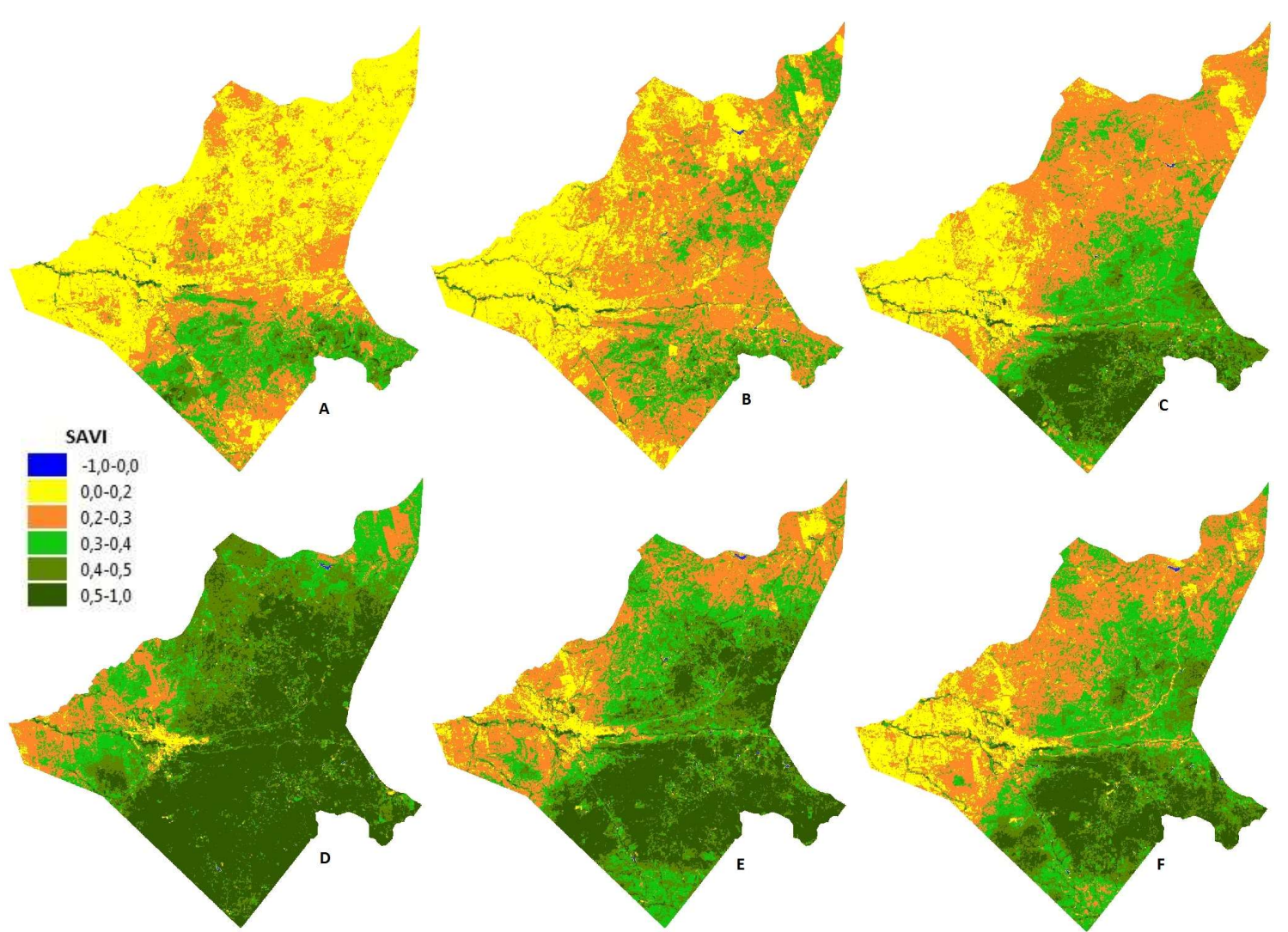

Figura 7. Variabilidade espaço-temporal do índice de vegetação SAVI nos dias 12/10/2003 (A), 18/09/2006 (B) (período seco); 19/07/2007 (C), 23/09/2008 (D) (período normal); 17/12/2004 (E) e 29/09/2010 (F) (período chuvoso) para o município de Arcoverde - PE.

Figure 7. Spatial-temporal variability of SAVI vegetation index in the days 10/12/2003 (A), 09/18/2006 (B) (dry period); 07/19/2007 (C), 09/23/2008 (D) (normal period); 12/17/2004 (E) and 09/29/2010 (F) (rainy season) for the municipality of Arcoverde - PE. 

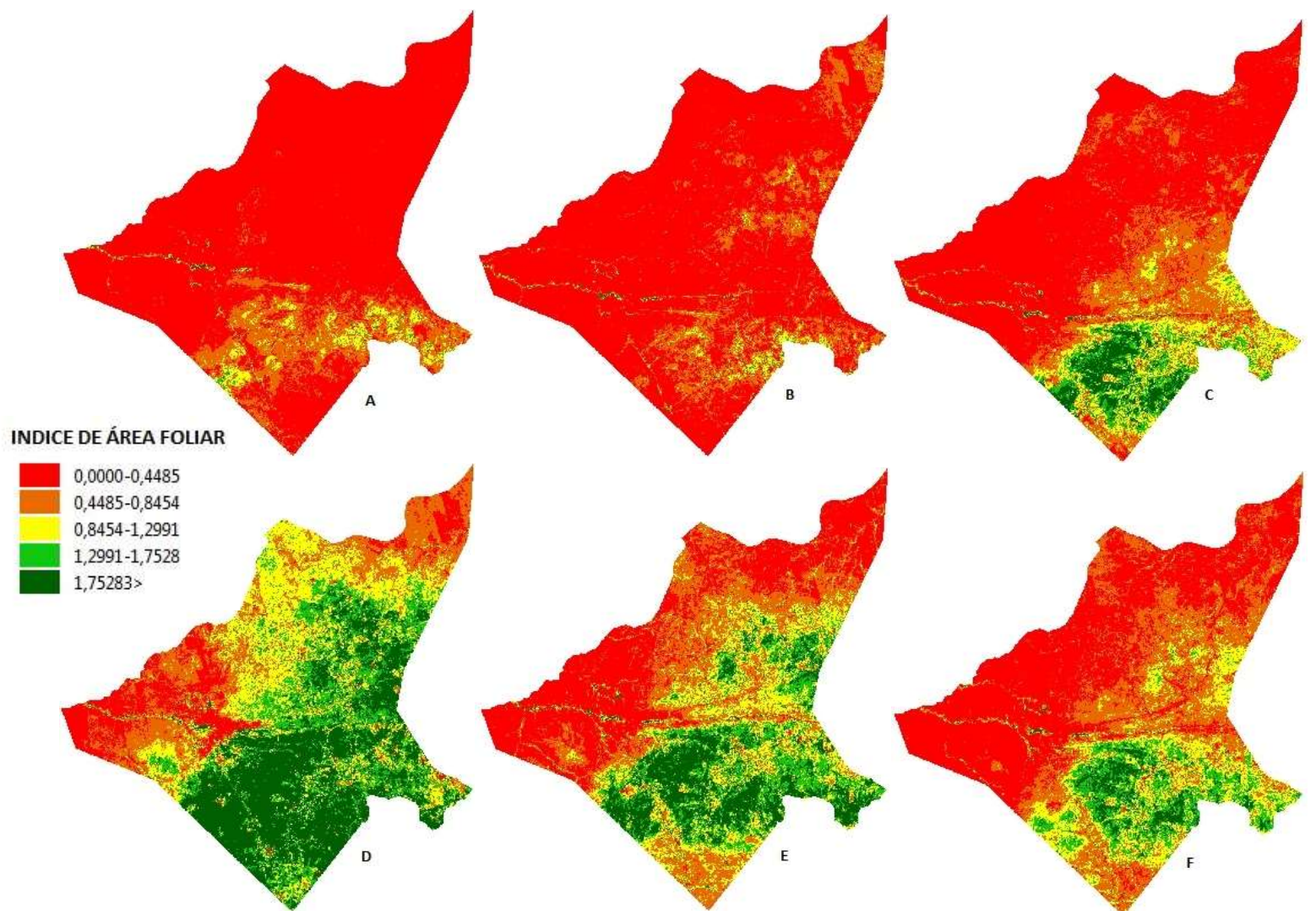

Figura 8. Variabilidade espaço-temporal do índice de vegetação IAF nos dias 12/10/2003 (A), 18/09/2006 (B) (período seco); 19/07/2007 (C), 23/09/2008 (D) (período normal); 17/12/2004 (E) e 29/09/2010 (F) (período chuvoso) para o município de Arcoverde - PE.

Figure 8. Spatial-temporal variability of IAF vegetation index in the days 10/12/2003 (A), 09/18/2006 (B) (dry period); 07/19/2007 (C), 09/23/2008 (D) (normal period); 12/17/2004 (E) and 09/29/2010 (F) (rainy season) for the municipality of Arcoverde - PE.

Tabela 3. Análise estatística do comportamento espaço-temporal dos índices de vegetação NDVI, SAVI e IAF para a região semiárida. Table 3. Statistical analysis of the spatial-temporal behavior of NDVI, SAVI and IAF vegetation indexes for the semiarid region.

\begin{tabular}{|c|c|c|c|c|c|c|c|c|}
\hline \multirow{2}{*}{$\begin{array}{c}\text { Classificação } \\
\text { dos anos }\end{array}$} & \multirow{2}{*}{$\begin{array}{l}\text { Índices de } \\
\text { vegetação }\end{array}$} & \multirow{2}{*}{ Data } & \multicolumn{6}{|c|}{ Parâmetros estatísticos } \\
\hline & & & Min & Max & Média & Moda & DP & CV (\%) \\
\hline \multirow{6}{*}{ Seco } & NDVI & \multirow{3}{*}{$12 / 10 / 2003$} & $-0,737$ & 0,834 & 0,269 & 0,199 & 0,11 & 41 \\
\hline & SAVI & & $-0,299$ & 0,787 & 0,224 & 0,170 & 0,09 & 40 \\
\hline & IAF & & 0,0 & 6,0 & 0,284 & 0,142 & 0,26 & 91 \\
\hline & NDVI & \multirow{3}{*}{$18 / 09 / 2006$} & $-0,710$ & 0,800 & 0,377 & 0,314 & 0,15 & 39 \\
\hline & SAVI & & $-0,396$ & 0,755 & 0,307 & 0,241 & 0,13 & 42 \\
\hline & IAF & & 0,0 & 6,0 & 0,574 & 0,283 & 0,54 & 94 \\
\hline \multirow{6}{*}{ Normal } & NDVI & \multirow{3}{*}{$19 / 07 / 2007$} & $-1,0$ & 0,852 & 0,574 & 0,706 & 0,15 & 26 \\
\hline & SAVI & & $-0,468$ & 0,794 & 0,481 & 0,584 & 0,13 & 27 \\
\hline & IAF & & 0,0 & 6,0 & 1,495 & 0,287 & 1,13 & 75 \\
\hline & NDVI & \multirow{3}{*}{$23 / 09 / 2008$} & $-1,0$ & 0,845 & 0,481 & 0,687 & 0,16 & 33 \\
\hline & SAVI & & $-0,382$ & 0,757 & 0,339 & 0,277 & 0,12 & 35 \\
\hline & IAF & & 0,0 & 6,0 & 0,957 & 0,307 & 0,73 & 76 \\
\hline \multirow{6}{*}{ Chuvoso } & NDVI & \multirow{3}{*}{$17 / 12 / 2004$} & $-1,0$ & 0,814 & 0,289 & 0,257 & 0,09 & 31 \\
\hline & SAVI & & 0,366 & 0,761 & 0,242 & 0,218 & 0,07 & 29 \\
\hline & IAF & & 0,0 & 6,0 & 0,322 & 0,236 & 0,23 & 71 \\
\hline & NDVI & \multirow{3}{*}{$29 / 09 / 2010$} & $-0,737$ & 0,807 & 0,414 & 0,378 & 0,15 & 36 \\
\hline & SAVI & & $-0,382$ & 0,757 & 0,339 & 0,277 & 0,12 & 35 \\
\hline & IAF & & 0,0 & 6,0 & 0,667 & 0,213 & 0,50 & 75 \\
\hline
\end{tabular}

\section{DISCUSSÃO}

Conforme os resultados dos parâmetros biofísicos mencionados anteriormente, o albedo foi um dos que destacou seus menores valores sobre superfícies de água e, também, sobre áreas bem vegetadas com forte presença da umidade (tonalidade azul claro e escuro). Os valores mais expressivos foram sobre a área urbana e, também, nas áreas com pouca e/ou nenhuma vegetação (pixel vermelho e marrom), o que podem indicar áreas em processo de degradação e/ou já degradadas na região (Figura 5). Lins et al. (2017) que também estudaram parâmetros biofísicos em região do semiárido brasileiro observaram altos valores de albedo sobre a área urbana do município, devido a maior refletividade. A área urbana da região é destacada em todos os mapas temáticos da área de estudo (Figura 5), localizada na região sudoeste.

Os dias 12/10/2003 e 18/09/2006, das respectivas Figuras $5 \mathrm{~A}$ e $5 \mathrm{~B}$, apresentam-se mais comumente com altos valores de albedo, evidenciando de fato o período de estiagem e a época seca que assola a região. Contudo, este parâmetro configurase num forte aliado para o monitoramento ambiental dos 
recursos hídricos e naturais do semiárido, principalmente na seca, contribuindo para o planejamento e tomadas de decisões sobre os diferentes usos do solo. Silva et al. (2005b) também estudando 0 albedo em região semiárida, destacaram a importância deste parâmetro neste sentido, por mostrar-se como excelente indicador nos estudos de mudanças climáticas e impactos ambientais. Silva et al. (2017) também em estudo sobre a região do semiárido brasileiro por sensoriamento remoto orbital, na bacia hidrográfica do Alto Ipanema destacaram que a dinâmica do padrão espacial do albedo da superfície está associada aos diferentes usos da terra e, principalmente, à vegetação heterogênea da Caatinga.

Em correspondência com o albedo, os mapas temáticos do índice de vegetação NDVI (Figura 6) apresentaram forte destaque sobre a época seca, confirmando o período de estiagem e seca da região nos anos de 2003 e 2006 (Figuras $6 \mathrm{~A}$ e $6 \mathrm{~B}$ ), com valores na faixa de zero e/ou próximos de zero. Com isso, observa-se que a época seca aferi negativamente o NDVI. Bezerra et al. (2014) destacaram que a análise espaçotemporal do NDVI está intimamente relacionada aos longos períodos de seca e a presença das ações antrópicas, que aceleram o processo da degradação ambiental e desertificação do solo no semiárido. Vale frisar também que as regiões semiáridas formadas por vegetação natural de Caatinga em períodos de estiagem e secas (déficit hídrico acentuado) se caracterizam pelas quedas das folhas, condição de defesa acentuado do bioma Caatinga (RODRIGUES et al., 2009; BEZERRA et al., 2014). Essa condição efetivamente, aumenta os valores do albedo e, consequentemente ocorre a diminuição do NDVI.

Os mapas dos períodos normal (Figuras 6C e 6D) e chuvoso (Figuras 6E, 6F) possuem maiores valores do NDVI, justificando a maior presença de umidade do solo na região, devido as precipitações antecedentes à passagem do satélite, visto que, os regimes de chuvas no ambiente semiárido de vegetação de Caatinga influenciam diretamente os índices de vegetação, sob condição de maiores valores do NDVI (RODRIGUES et al., 2009; ARRAES et al., 2012; BEZERRA et al., 2014). Este comportamento corrobora ao observado no estudo de Aquino; Oliveira (2012), também em região semiárida, onde no período chuvoso houve uma redução das áreas de solo exposto, configurando-se no aumento do NDVI, justificado em função do aumento das precipitações e, consequentemente pelo aparecimento de espécies vegetais. Também em estudo no semiárido brasileiro, Ribeiro et al. (2016) destacaram que o NDVI mostrou o poder de resiliência da Caatinga através do elevado potencial de recuperação e desenvolvimento de biomassa em condições favoráveis de precipitação.

As mudanças da superfície terrestre se mostraram com mais acurácia no índice de vegetação SAVI (Figura 7), destacando melhor a resposta espectral da vegetação, e ainda, apresentando mais claramente no período seco a influência de solo exposto. O SAVI apresenta assim, uma dinâmica espaçotemporal da vegetação natural de Caatinga sob condições próximas as reais existentes na área de estudo, pois este parâmetro busca amenizar os efeitos "background" do solo (RIBEIRO et al., 2016). Estudos realizados no semiárido brasileiro corroboram a estas informações, e destacam que o índice de vegetação SAVI possui mais acurácia nos estudos da vegetação de Caatinga, principalmente no período seco dessas regiões (SILVA; GALVÍNCIO, 2012; OLIVEIRA et al., 2017).
Corroborando ao presente estudo, Silva; Galvíncio (2012) diante de pesquisa desenvolvida também em região do semiárido brasileiro a partir da comparação dos índices de vegetação NDVI e SAVI, observaram maior eficácia no SAVI, o qual destacou-se principalmente no período seco, confirmando sua alta aplicabilidade e confiabilidade para estudos da vegetação de Caatinga no semiárido.

O IAF destaca em todos os mapas a forte ausência de área foliar na região (Figura 8), que mostrou ser afetado excepcionalmente pelo período de estiagem e seca (mapas das Figuras 8A e 8B), e ainda, considerando principalmente também, os períodos analisados nos anos classificados climaticamente como normais (mapas das Figuras 8C e 8D) e chuvosos (mapas das Figuras 8E e 8F), com baixos valores de IAF. Todavia, podem ser indícios preocupantes de áreas degradadas ou em processo de degradação, bem como, a possibilidade da presença de áreas desertificadas. Apesar que nestes períodos (normal e chuvoso) também foram observados altos valores de IAF, justificado possivelmente pela presença de disponibilidade hídrica.

Nesse sentido, além da estiagem, o decréscimo nos valores do IAF pode ser explicado também pela maior atividade agrícola na área de estudo, motivado através do desmatamento da cobertura vegetal.

Em geral, os índices de vegetação apresentaram condições de distribuição semelhantes, principalmente para o período seco da análise espaço-temporal, nos dias 12/10/2003 (A) e 18/09/2006 (B), justificando este período com baixos valores dentre todos os índices, situando-se na faixa de zero e/ou próximos de zero (valores positivos), indicando de fato as áreas mais afetadas pela seca na região semiárida. Deste modo, todos os parâmetros analisados como, albedo e os índices de vegetação supracitados identificaram possivelmente áreas degradadas ou em processo de degradação, localizados principalmente a sudoeste e noroeste da localidade de Arcoverde-PE. A área de estudo apresentou altos valores de albedo ao passo que os índices de vegetação NDVI, SAVI e IAF foram significativamente menores.

Rêgo et al. (2012) também identificaram através de índices de vegetação o crescimento de áreas com nível de degradação considerado grave em região localizada também no semiárido brasileiro, destacando ao longo dos anos estudados o aumento do processo de desertificação na região, motivados pelos longos períodos de estiagem e seca, e também pelas atividades antrópicas. Vale destacar que os estudos sobre fatores ligados à vegetação possuem ampla influência nos processos do balanço de radiação à superfície e evapotranspiração (SILVA et al., 2005a).

Conforme a Tabela 3, os períodos seco, normal e chuvoso apresentaram uma distribuição espaço-temporal para o NDVI e SAVI de média variabilidade (CV entre 26 e 42\%). O IAF apresentou-se de alta variabilidade (CV entre 75 e 91\%) (WARRICK; NIELSEN, 1980).

Avaliando as médias dos anos classificados como secos (anos de 2003 e 2006), o dia 12/10/2003 apresentou médias de NDVI, SAVI e IAF inferiores ao dia de 18/09/2006 (Tabela 3), mesmo com maior precipitação acumulada e distribuída durante o ano, onde no trimestre anterior foi de $158,7 \mathrm{~mm} \mathrm{e}$ um total médio anual de 474,7 mm (Figura 2 e Tabela 1), tal fato pode ser explicado pelo longo período de estiagem e seca na região, que durou do ano de 1998 a 2003 conforme registros do INMET, com exceção do ano de 2000 (classificado como normal), favorecendo ao efeito de déficit hídrico para as 
plantas na região e, consequentemente prejudicando seu desenvolvimento, o que justifica os baixos valores nos índices de vegetação para o dia 12/10/2003 quando comparado ao dia 18/09/2006. Mariano et al. (2018) destacaram que o agravamento da degradação do solo nas regiões semiáridas é um dos resultados mais catastróficos de eventos de seca de longa duração e atividades antrópicas.

Analisando também as médias dos índices de vegetação dos anos classificados climaticamente como normais (anos de 2007 e 2008), o dia 19/07/2007 apresentou valores de NDVI, SAVI e IAF superior ao dia 23/09/2008 (Tabela 3). Isso mostra que os índices de vegetação foram influenciados diretamente pela maior precipitação ocorrida no trimestre anterior $(275,5$ $\mathrm{mm}$ ) ao imageamento do dia 19/07/2007 e, ainda, teve uma maior precipitação média anual de $835,7 \mathrm{~mm}$ (Figura $3 \mathrm{e}$ Tabela 1). Rodrigues et al. (2009) também observaram altos valores de índice de vegetação em região do semiárido brasileiro, influenciado pelas precipitações antecedentes a passagem do satélite no período chuvoso da região.

Ainda na Tabela 3, as médias dos índices classificados climaticamente como chuvosos (anos de 2004 e 2010), o dia 29/09/2010 apresentou valores médios dos índices de vegetação bem superiores ao dia 17/12/2004, influenciados também pela maior precipitação observada no trimestre anterior $(298,9 \mathrm{~mm})$ ao imageamento pelo satélite e pela precipitação média anual maior, de 977,2 mm (Figura 4 e Tabela 1), corroborando as observações de Arraes et al. (2012), em região semiárida, que identificaram sobre áreas de vegetação nativa de Caatinga, os maiores valores do índice de vegetação NDVI, sendo na época do período chuvoso, onde geralmente a vegetação tem presença expressiva no semiárido, motivados pelos regimes de chuva, tal qual favorece ao maior aporte de umidade, contribuindo diretamente para ascensão dos índices de vegetação.

\section{CONCLUSÕES}

Os índices de vegetação destacaram-se como bons indicadores de áreas em processo de degradação e/ou possivelmente já degradadas, indicando claramente modificações ambientais sobre o uso e ocupação do solo da região semiárida, motivados principalmente pela seca e/ou ações antrópicas, como atividades agrícolas, que envolve o desmatamento de áreas vegetadas.

O SAVI mostrou-se mais adequado dentre os índices de vegetação, permitindo destacar a dinâmica espaço-temporal da vegetação de Caatinga na região semiárida com maior precisão, minimizando os efeitos atmosféricos e do solo, favorecendo assim, a uma resposta espectral da vegetação com mais acurácia.

O período classificado climaticamente como seco foi nitidamente destacado pelo albedo e os índices de vegetação NDVI, SAVI e IAF através do monitoramento ambiental por satélite, permitindo afirmar que o sensoriamento remoto é um forte aliado para estudos principalmente em épocas de estiagem e seca, períodos de alto risco de degradação e desertificação, contribuindo para o planejamento e na tomada de decisões sobre os recursos hídricos e naturais, visando minimizar os impactos ambientais no semiárido brasileiro.

\section{AGRADECIMENTOS}

Ao Programa de Pós-Graduação em Engenharia Agrícola; Universidade Federal Rural de Pernambuco; CAPES e CNPq pelas bolsas PIBIC e de Doutorado; Ao INMET e APAC.

\section{REFERÊNCIAS}

ALLEN, R. G.; TASUMI, M.; TREZZA, R. Satellite-Based energy balance for mapping evapotranspiration with internalized calibration (METRIC) - Model. Journal of Irrigation and Drainage Engineering, New York, v. 133, p. 380-394, 2007.

ALLEN, R. G.; TASUMI, M.; TREZZA, R.; BASTIAANSSEN, W. G. M. SEBAL (Surface Energy Balance Algorithms for Land) - Advance Training and Users Manual-Idaho Implementation. 2002. 1 v. 97 p.

ALVARES, C. A.; STAPE, J. L.; SENTELHAS, P. C.; MORAES, G. de; LEONARDO, J.; SPAROVEK, G. Köppen's climate classification map for Brazil. Meteorologische Zeitschrift, Berlin, v. 22, p. 711-728, 2013. DOI: https://dx.doi.org/10.1127/09412948/2013/0507

AQUINO, C. M. S.; OLIVEIRA, J. G. B. Estudo da dinâmica do índice de vegetação por diferença normalizada (NDVI) no núcleo de São Raimundo Nonato-PI. GEOUSP: Espaço e Tempo (Online), São Paulo, n. 31, p. 157-168. 2012.

ARRAES, F. D. D.; ANDRADE, E. M.; SILVA, B. B. Dinâmica do balanço de energia sobre o açude Orós e suas adjacências. Caatinga, Mossoró, v. 25, p. 119-127, 2012.

BASTIAANSSEN, W. G. M. SEBAL-based sensible and latent heat fluxes in the irrigated Gediz Basin, Turkey. Journal of Hydrology, Amsterdam, v. 229, n. 1-2, p. 87100, 2000. DOI: https://dx.doi.org/10.1016/S00221694(99)00202-4

BELTRÃO, B. A.; MASCARENHAS, J. C.; MIRANDA, J. L. F.; SOUZA JUNIOR, L. C.; GALVÃO, M. J. T. G.; PEREIRA, S. N. Projeto cadastro de fontes de abastecimento por água subterrânea no estado de Pernambuco - Diagnóstico do município de Arcoverde. Recife: CPRM/PRODEEM, 2005. $11 \mathrm{p}$.

BEZERRA, J. M.; MOURA, G. B.; SILVA, B. B.; LOPES, P. M.; SILVA, Ê. F. F. Parâmetros biofísicos obtidos por sensoriamento remoto em região semiárida do estado do Rio Grande do Norte, Brasil. Revista Brasileira de Engenharia Agrícola e Ambiental, Campina Grande, v. 18 , n. 1, p. 73-84, 2014. DOI: https://dx.doi.org/10.1590/s1415-43662014000100010

CHANDER, G.; MARKHAM, B. L.; HELDER, D. L. Summary of current radiometric calibration coefficients for Landsat MSS, TM, ETM+, and EO-1 ALI sensors. Remote Sensing of Environment, New York, v. 113, n. 5, p. 893-903, 2009. DOI: https://dx.doi.org/10.1016/j.rse.2009.01.007

GARRISON, J. D.; ADLER, G. P. Estimation of precipitable water over the United State for application to the division of solar radiation into its direct and diffuse components. Solar Energy, Kidlington v. 44, n. 4, p. 225-241, 1990. DOI: https://dx.doi.org/10.1016/0038-092X(90)90151-2

GIONGO, P. R.; VETTORAZZI, C. A. Albedo da superfície por meio de imagens TM-Landsat 5 e modelo numérico do terreno. Revista Brasileira de Engenharia Agrícola e Ambiental, Campina Grande, v. 18, n. 8, p. 833-838, 2014. DOI: http://dx.doi.org/10.1590/18071929/agriambi.v18n08p833-838

HUETE, A. R. A soil-adjusted vegetation index (SAVI). Remote Sensing of Environment, New York, v. 25, n. 3, p. 295-309, 1988. DOI: https://dx.doi.org/10.1016/00344257(88)90106-X 
IBGE INSTITUTO BRASILEIRO DE GEOGRAFIA E ESTATÍSTICA. Divisão Territorial do Brasil e Limites Territoriais. Disponível em: $<\mathrm{ftp}$ //geoftp.ibge.gov.br/organizacao_do_territorio/estrut ura_territorial/divisao_territorial $/ 2016 />$ Acesso: $18 \mathrm{de}$ setembro de 2018

LINS, F. A. C.; ARAÚJO, D. C. S.; SILVA, J. L. B.; LOPES, P. M. O.; OLIVEIRA, J. D. A.; SILVA, A. T. C. S. G. Estimativa de parâmetros biofísicos e evapotranspiração real no semiárido Pernambucano utilizando sensoriamento remoto. Irriga, Botucatu, v. 1, n. 1, p. 64-75, 2017. DOI: https://dx.doi.org/10.15809/irriga.2017v1n1p64-75

MARIANO, D. A.; SANTOS, C. A. dos; WARDLOW, B. D.; ANDERSON, M. C.; SCHILTMEYER, A. V.; TADESSE, T.; SVOBODA, M. D. Use of remote sensing indicators to assess effects of drought and human-induced land degradation on ecosystem health in Northeastern Brazil. Remote Sensing of Environment, New York, v. 213, p. 129-143, 2018.

DOI: https://dx.doi.org/10.1016/j.rse.2018.04.048

MMA MINISTÉRIO DO MEIO AMBIENTE. Caatinga. 2018. Disponível em $<$ http://www.mma.gov.br/biomas/caatinga>. Acesso em 07 de dezembro de 2018.

MMA MINISTÉRIO DO MEIO AMBIENTE (MMA). Monitoramento do desmatamento nos biomas brasileiros por Satélite: Monitoramento do bioma Caatinga 2002-2009. Acordo de cooperação técnica MMA/IBAMA - Instituto Brasileiro do Meio Ambiente e dos Recursos Naturais Renováveis. Brasília/DF. 2011. Disponível

em: $<$ http://siscom.ibama.gov.br/monitora_biomas/PMDBBS $\% 20-\% 20$ CAATINGA.html>. Acesso em 17 de setembro de 2018 .

OLIVEIRA, J. D. A.; MEDEIROS, B. C.; SILVA, J. L. B.; ALBUQUERQUE MOURA, G. B.; LINS, F. A. C.; NASCIMENTO, C. R.; LOPES, P. M. O. Space-temporal evaluation of biophysical parameters in the High Ipanema watershed by remote sensing. Journal of Hyperspectral Remote Sensing, Recife, v. 7, n. 6, p. 357-366, 2017.

OLIVEIRA, S. B. P.; MARTINS, E. S. P. R.; CARVALHO, M. S. B. D. S.; SIFEDINE, A.; FERRAZ, B. Uso de sensoriamento remoto para mapeamento de áreas susceptíveis à desertificação na região semiárida do Brasil. Ciência \& Trópico, Recife, v. 41, p. 67-96, 2017.

RÊGO, S. C. A.; LIMA, P. P. S.; LIMA, M. N. S.; MONTEIRO, T. R. R. Análise comparativa dos índices de vegetação NDVI e SAVI no município de São Domingos do Cariri-PB. Revista geonorte, Manaus, v. 3, n. 5, p. 1217-1229, 2012

RIBEIRO, E. P.; NÓBREGA, R. S.; MOTA FILHO, F. O.; MOREIRA, E. B. Estimativa dos índices de vegetação na detecção de mudanças ambientais na bacia hidrográfica do rio Pajeú. Geosul, Florianópolis, v. 31, n. 62, p. 59-92, 2016.

RODRIGUES, J. D.; ANDRADE, E. D.; TEIXEIRA, A. D. S.; SILVA, B. D. Sazonalidade de variáveis biofísicas em regiões semiáridas pelo emprego do sensoriamento remoto. Engenharia Agrícola, Jaboticabal, v. 29, n. 3, p. 452-465, 2009. DOI: http://dx.doi.org/10.1590/S010069162009000300012

SILVA, B. B. D.; BRAGA, A. C.; BRAGA, C. C.; OLIVEIRA, L. M.; MONTENEGRO, S. M.; BARBOSA
JUNIOR, B. Procedures for calculation of the albedo with OLI-Landsat 8 images: Application to the Brazilian semiarid. Revista Brasileira de Engenharia Agrícola e Ambiental, v. 20, n. 1, p. 3-8, 2016. DOI: http://dx.doi.org/10.1590/1807-1929/agriambi.v20n1p3-8

SILVA, B. B.; LOPES, G. M.; AZEVEDO, P. V. Balanço de radiação em áreas irrigadas utilizando imagens Landsat 5 - TM. Revista Brasileira de Meteorologia, São José dos Campos, v. 20, n. 2, p. 243-252, 2005a.

SILVA, B. B.; LOPES, G. M.; AZEVEDO, P. V. Determinação do albedo de áreas irrigadas com base em imagens Landsat 5 TM. Revista Brasileira de Agrometeorologia, v. 13, p. 201-211. 2005b.

SILVA, J. L. B.; SANTOS ARAÚJO, D. C.; SANTOS, D. P.; BEZERRA, A. C.; ALBUQUERQUE MOURA, G. B.; OLIVEIRA, P. M. L.; SILVA, H. P. Spatial distribution of biophysical parameters in the Ipanema River Basin using remote sensing techniques. Journal of Hyperspectral Remote Sensing, Recife, v. 7, n. 6, p. 324-333, 2017.

SILVA, L. G.; GALVÍNCIO, J. D. Análise comparativa da variação nos índices NDVI e SAVI no Sítio PELD - 22, em Petrolina - PE, na primeira década do século XXI. Revista Brasileira de Geografia Física, Recife, v. 5, n. 6, p. 1446-1456, 2012.

WARRICK, A. W.; NIELSEN, D. R. Spatial variability of soil physical properties in the field. In: HILLEL, D. (Ed.) Applications of soil physics. New York: Academic Press. 1980. p. 319-344.

WWF WORLD WIDE FUND FOR NATURE. As Müdanças Climáticas. Disponível em: $<$ http://www.wwf.org.br/natureza_brasileira/reducao_de impactos2/clima/mudancas_climaticas $2 />$. Acesso: $17 \mathrm{de}$ setembro de 2018.

XAVIER, T. M. B. S.; XAVIER, A. F. S. Caracterização de períodos secos ou excessivamente chuvosos no estado do Ceará através da técnica dos Quantis: 1964-1998. Revista Brasileira de Meteorologia, São José dos Campos, v. 14, n. 2, p. 63-78, 1999. 